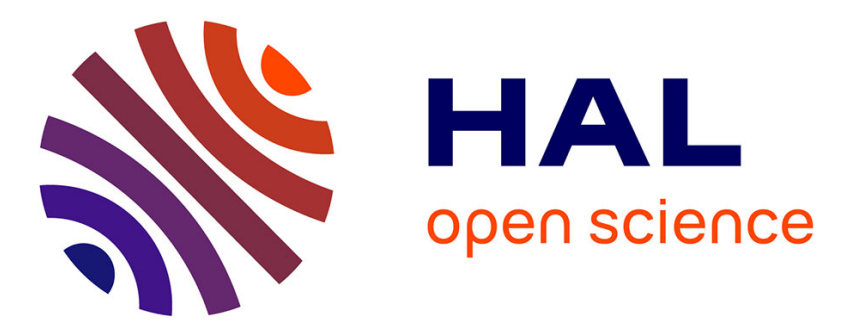

\title{
Local Mixing Events in the Upper Troposphere and Lower Stratosphere. Part II: Seasonal and Interannual Variability
}

Emily Shuckburgh, Francesco d'Ovidio, Bernard Legras

\section{- To cite this version:}

Emily Shuckburgh, Francesco d'Ovidio, Bernard Legras. Local Mixing Events in the Upper Troposphere and Lower Stratosphere. Part II: Seasonal and Interannual Variability. Journal of the Atmospheric Sciences, 2009, 66, pp.3695. 10.1175/2009JAS2983.1 . hal-00763159

\section{HAL Id: hal-00763159 \\ https://hal.science/hal-00763159}

Submitted on 22 Oct 2021

HAL is a multi-disciplinary open access archive for the deposit and dissemination of scientific research documents, whether they are published or not. The documents may come from teaching and research institutions in France or abroad, or from public or private research centers.
L'archive ouverte pluridisciplinaire HAL, est destinée au dépôt et à la diffusion de documents scientifiques de niveau recherche, publiés ou non, émanant des établissements d'enseignement et de recherche français ou étrangers, des laboratoires publics ou privés.

\section{(c)(1)}

Distributed under a Creative Commons Attribution| 4.0 International License 


\title{
Local Mixing Events in the Upper Troposphere and Lower Stratosphere. Part II: Seasonal and Interannual Variability
}

\author{
EMILY SHUCKBURGH \\ British Antarctic Survey, Cambridge, United Kingdom \\ FRANCESCO D'OVIDIO \\ Laboratoire de Météorologie Dynamique, Ecole Normale Supérieure, and ISC-PIF, Paris, France \\ BERNARD LEGRAS \\ Laboratoire de Météorologie Dynamique, Ecole Normale Supérieure, and CNRS, Paris, France
}

(Manuscript received 16 October 2008, in final form 5 June 2009)

\begin{abstract}
The Lyapunov diffusivity is used to investigate local isentropic mixing events in the upper tropospherelower stratosphere (UTLS) region. The diagnostic highlights the seasonal cycle of the longitudinally varying mixing properties, in particular those associated with the monsoon circulations and the westerly ducts in the subtropics. The results are broadly consistent with studies of Rossby wave-breaking frequencies. The mixing structure is shown to be modulated by modes of atmospheric variability. El Niño-Southern Oscillation (ENSO) is found to strongly influence the mixing structure throughout the tropics and subtropics. The subtropical jet is associated with longitudinal bands of mixing minima (isentropic mixing barriers) separated by synoptic-scale regions of strong mixing activity. The greatest ENSO modulation in December-February is confined to the Pacific sector, where the barriers associated with the subtropical jets extend farther into the eastern Pacific, and in the western Pacific a barrier is found at the equator during the positive phase. During June-August, the influence is seen to extend beyond the Pacific region, with the barrier at the subtropical jet in the Southern Hemisphere increasing in strength at all longitudes and with an increase in strength (and isolation of) monsoon-related mixing over Asia and North America. The local influence of the North Atlantic Oscillation in wintertime is investigated. During the positive phase, a double-barrier structure is associated with the subtropical jet, the northern branch crosses the Atlantic toward Scandinavia, and the southern branch tends toward North Africa. The Antarctic Oscillation is shown to influence whether the subvortex region is isolated from midlatitudes.
\end{abstract}

\section{Introduction}

Mixing along isentropic surfaces that intersect the tropopause provides an important pathway for the exchange of chemical species between the troposphere and the stratosphere. Adiabatic eddy motions along the isentropes can lead to large latitudinal displacements of the tropopause, and transport of species follows by irreversible mixing on small scales. It is often useful to distinguish this isentropic eddy transport from cross-

Corresponding author address: Emily Shuckburgh, British Antarctic Survey, High Cross, Madingley Rd., Cambridge CB3 0ET, United Kingdom.

E-mail: emsh@bas.ac.uk isentropic transport, which involves different dynamical processes and different time scales (Holton et al. 1995). The distribution of the mixing ratio of long-lived chemical species on such cross-tropopause isentropic surfaces (e.g., $\mathrm{N}_{2} \mathrm{O}$; Hegglin et al. 2006; Strahan et al. 2007) shows strong latitudinal gradients in the vicinity of the subtropical jet. This is indicative of an inhibition of isentropic eddy transport at that location. Nevertheless, some mixing across the subtropical jet does occur, as is evident from the mixing lines seen in the correlations between tracers whose lifetimes in the upper troposphere-lower stratosphere (UTLS) region are sufficiently long to be affected by transport (e.g., $\mathrm{O}_{3}-\mathrm{CO}$ and $\mathrm{O}_{3}-\mathrm{H}_{2} \mathrm{O}$; Hoor et al. 2002; Pan et al. 2007; Hegglin et al. 2008). In addition, observational and modeling studies (e.g., Stohl et al. 
2003) indicate that there are preferred geographical locations (in longitude and latitude) for stratospheretroposphere exchange. Broadly, the transport and mixing structure can be understood in terms of the potential vorticity (PV) distribution (for a review, see McIntyre 2008, and references therein). When the background PV gradient is small, large-amplitude Rossby waves may break and result in mixing (of PV and trace constituents) over a longitudinally confined region. However, when the PV gradients are large, associated with strong jets in the flow, the Rossby wave restoring mechanism inhibits transport (for an example of this effect in a simple dynamically consistent PV-conserving flow, see Haynes et al. 2007). Seasonal differences in the chemical composition of the lowermost stratosphere (see also, e.g., Hegglin et al. 2006; Strahan et al. 2007) suggest that there are seasonal differences in the strength of the isentropic transport and mixing in the tropopause region.

Haynes and Shuckburgh (2000b) used the effective diffusivity diagnostic (Nakamura 1996) to quantify the isentropic mixing and transport in the upper troposphere and lower stratosphere generated by analyzed winds from the European Centre for Medium-Range Weather Forecasts (ECMWF). Their results demonstrated that the subtropical jets are locations of longitudinal minima in large-scale stirring; thus, they do indeed act as a barrier to eddy transport, with significantly reduced eddy-induced mixing compared to the extratropics (the effective diffusivity differing by a factor of 4 or more) throughout much of the year. A distinct seasonal cycle was identified with a stronger barrier to transport at the subtropical jet in winter than in summer, which is consistent with observations of chemical species. However, a critical limitation of the effective diffusivity diagnostic is that it cannot provide information about the longitudinal variations in transport and mixing. By construct, it provides information averaged around a contour of equivalent latitude $\phi_{e}$. To address this problem, we introduced a new diagnostic of transport and mixing, the Lyapunov diffusivity, in the first part of this study (d'Ovidio et al. 2009, hereafter Part I). The diagnostic takes the effective diffusivity and exploits information from Lyapunov exponents and vectors to resolve the local mixing fluctuations along an equivalent latitude. In this way, it is able to provide a complete latitude-longitude description of the isentropic transport and mixing structure.

Part I presented the annual mean Lyapunov diffusivity for the $350-\mathrm{K}$ isentropic surface, ${ }^{1}$ calculated from

\footnotetext{
${ }^{1}$ The 350-K surface lies in the "middleworld" (Hoskins 1991), where isentropes cross the tropopause but do not intersect the earth's surface.
}

40-yr ECMWF Re-Analysis (ERA-40) wind data from the time period 1981-2001. Significant longitudinal variability was observed, in particular in the strength of bands of minima in isentropic mixing (that we will call "mixing barriers" in the following) at the subtropical jet. These structures were seen to be strongest on the western sides of the Pacific and Atlantic, and to be interrupted by synoptic-scale systems, which can induce strong isentropic mixing. Here we examine in detail interannual variations to these transport and mixing structures and their relation to known modes of climate variability such as El Niño-Southern Oscillation (ENSO) and the North Atlantic Oscillation (NAO). In particular we show quantitatively how a "duct" in the subtropical jet barrier opens and closes according to ENSO cycle, modulating exchanges between tropical and extratropical latitudes.

This paper is organized in the following manner: in section 2 , we briefly revisit the data and methodology used in Part I to calculate the Lyapunov diffusivities. Then, in section 3, we investigate the seasonal variability of the Lyapunov diffusivity on the $350-\mathrm{K}$ isentropic surface. In section 4 , we consider the influence of modes of atmospheric variability on the transport and mixing structure, focusing on El Niño-Southern Oscillation, the North Atlantic Oscillation, and the Antarctic Oscillation. Finally, we summarize our results and suggest further applications in section 5 .

\section{Data and method}

A new diagnostic of transport and mixing was presented in Part I: the Lyapunov diffusivity. The diagnostic is based on information coming from two separate calculations: a tracer-based calculation giving the effective diffusivity (Nakamura 1996; Haynes and Shuckburgh 2000a; Shuckburgh and Haynes 2003) and a particle-based calculation giving the Lyapunov exponents and vectors (e.g., Joseph and Legras 2002; Koh and Legras 2002; d'Ovidio et al. 2004). The new diagnostic uses local information concerning the stretching intensity (from the Lyapunov exponents) and geometry (from the Lyapunov vectors), along with the effective diffusivity. The construction of the diagnostic has been guided by theoretical arguments, as described in Part I.

Both the Lyapunov exponent/vector calculation and the effective diffusivity calculation are based on twodimensional velocity fields. Here, we consider isentropic velocities on the $350-\mathrm{K}$ isentropic surface from the ERA-40 dataset provided by the European Centre for Medium-Range Weather Forecasts (Uppala et al. 2005) over the period 1981-2001. The choice of this period is dictated by the relative homogeneity of the assimilated data, notably the satellite data provided by Television 
and Infrared Observation Satellite (TIROS) Operational Vertical Sounder (TOVS) instruments, cloudwind products, and surface data from buoys. The two diagnostics are computed every two days for the entire period, according to the methods described in Part I.

The Lyapunov diffusivity $D_{\lambda}$, a function of longitude, latitude, and time, is defined by the following:

$$
\begin{aligned}
D_{\lambda}(\theta, \phi, t):= & \kappa_{a}\left(\phi_{e}\right)+b\left(\phi_{e}\right) L_{\text {eddy }}^{2}(\theta, \phi, t) \\
& \times \lambda(\theta, \phi, t) \sin ^{2} \alpha,
\end{aligned}
$$

where $\phi_{e}(\theta, \phi, t)$ is the equivalent latitude, $L_{\text {eddy }}$ is a length scale representing the most energetic perturbations, $\lambda$ is the finite-size Lyapunov exponent calculated forward in time, and $\alpha$ is the angle between the forward and backward Lyapunov vectors. The coefficients $\kappa_{a}$ and $b$ are obtained by a linear fit process, taking the along-equivalent-latitude average of Eq. (1) and setting it equal to the effective diffusivity.

The recipe for the calculation of the Lyapunov diffusivity is the following: at each grid point (in longitude, latitude, and time) compute forward and backward Lyapunov exponents and vectors using the velocity field. Then, again at each grid point, calculate the angle between forward and backward principal Lyapunov vectors and use it to obtain the modified Lyapunov exponent $\lambda \sin ^{2} \alpha$ by multiplying the forward Lyapunov exponent by the geometric factor. Multiply this by the length scale squared of the most energetic perturbations (we take this to be $1000 \mathrm{~km}$, decreasing as sine of latitude at high latitudes to represent the decrease in size of the Rossby deformation radius). Next, calculate the effective diffusivity using the velocity field together with a probe tracer. Take the average along each equivalent latitude of $L_{\text {eddy }}^{2} \lambda \sin ^{2} \alpha$, and then use a linear fit with the effective diffusivity to obtain the two (equivalent-latitude dependent) coefficients $\kappa_{a}$ and $b$. Finally, use the estimated coefficients for computing the Lyapunov diffusivity with Eq. (1).

The calculation is somewhat laborious, including both particle and tracer integrations. However, the results provide, for the first time, a comprehensive quantification of the latitude-longitude distribution of mixing in the UTLS in units of diffusion. The results can be used to interpret observational data and to validate chemistryclimate models.

\section{A climatology of local mixing at $350 \mathrm{~K}$}

The effective diffusivity for the mean annual cycle of the UTLS has been analyzed previously by Haynes and Shuckburgh (2000b). Here, we focus on the latitudinal and longitudinal variability of isentropic mixing at $350 \mathrm{~K}$ revealed by the Lyapunov diffusivity. Figure 1 presents the Lyapunov diffusivity as a gridded map for each of the four seasons, averaged over the period 1981-2001. At all latitudes, local mixing regions may be characterized by intensities several times stronger than their zonal mean. As noted in Part I, both hemispheres are characterized by mixing barriers at the subtropical jets. The strongest barrier is located over Asia and the western Pacific in the Northern Hemisphere and over Australia in the Southern Hemisphere.

The pattern of mixing intensity is strongly modulated by the annual cycle, with a dramatic switch occurring during late spring and early autumn. During DecemberFebruary (DJF; Fig. 1a) a strong barrier exists associated with the northern subtropical jet (see also Fig. 2). This barrier is strongest where the jet velocities are strongest over the western Pacific (less than $10^{5} \mathrm{~m}^{2} \mathrm{~s}^{-1}$; dashed line in Fig. 2) and western Atlantic. Mixing regions are found in the jet exit regions in the eastern Pacific and eastern Atlantic $\left(\sim 10^{6} \mathrm{~m}^{2} \mathrm{~s}^{-1}\right.$; dotted and dashed lines in Fig. 2, respectively). These mixing regions are downstream of the regions of largest baroclinicity (see, e.g., Hoskins and Valdes 1990). This highlights the fact that local eddy kinetic energy cannot be used alone to parameterize mixing; the mean flow needs to be taken into account as well (Shuckburgh et al. 2009a,b). Over Asia and the Pacific, the strongest barrier is located at $\sim 25^{\circ} \mathrm{N}$, with a broad, weaker barrier from $15^{\circ}$ to $45^{\circ} \mathrm{N}$. Over the eastern Atlantic and into Europe, the region from $15^{\circ}$ to $45^{\circ} \mathrm{N}$ is represented by weak mixing, with a narrow, weak barrier following the streamlines to the south, located at $\sim 20^{\circ} \mathrm{N}$. On individual days (not shown) there appear to be two distinct barriers following the streamlines: one around $20^{\circ} \mathrm{N}$ and the other around $45^{\circ} \mathrm{N}$. This structure may be representative of a barrier at the weak subtropical jet and a second eddy-driven jet some $20^{\circ}$ poleward (Lee and Kim 2003), with weak mixing between. Poleward of the subtropical jet is a region of weak-moderate mixing, which extends all the way to the pole, allowing transport into the subvortex region, which is consistent with aircraft observations of chemical species (Spackman et al. 2007; Ziereis et al. 2000).

There is a weak barrier at the subtropical jet in the Southern Hemisphere, centered on about $45^{\circ} \mathrm{S}$, with the strongest barrier being located in the Atlantic and Indian Ocean sectors. The break in the barrier across the Pacific corresponds to a region of high wave-breaking frequency (Postel and Hitchman 1999; Berrisford et al. 2007). Poleward of this, there is a region of enhanced mixing along the coast of Antarctica that also corresponds to a region of high wave-breaking frequency (Berrisford et al. 2007). The magnitude of the diffusivities in the region of the Southern Hemisphere subtropical jet (about $10^{6} \mathrm{~m}^{2} \mathrm{~s}^{-1}$ ) are comparable to the diffusivities in the 
DJF 350K

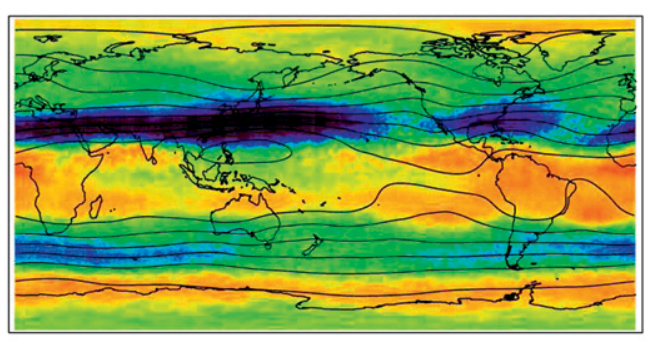

MAM 350K

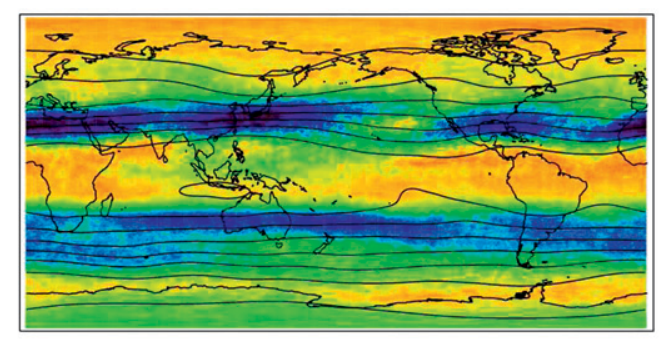

JJA $350 \mathrm{~K}$

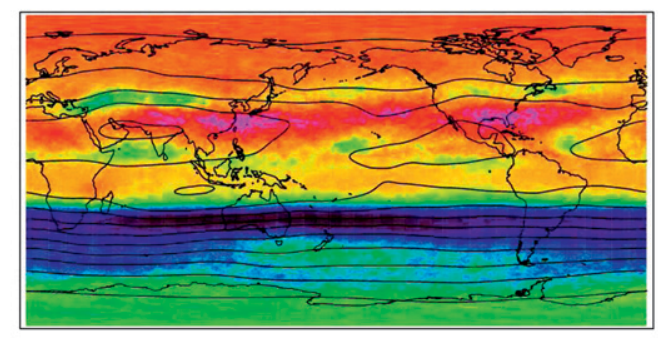

SON 350K

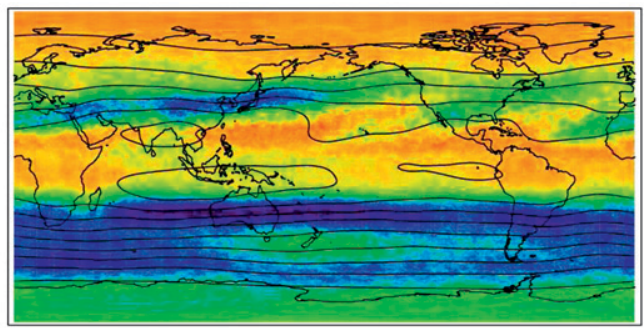

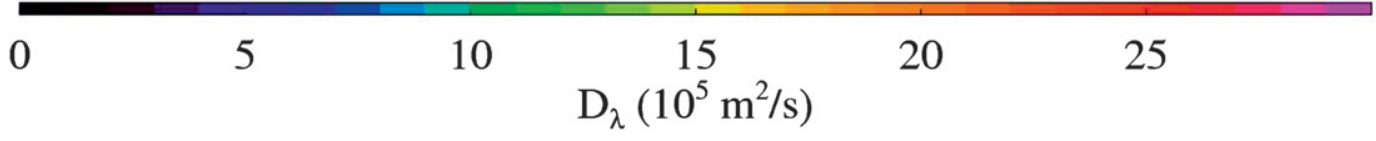

FIG. 1. Seasonal cycle of the Lyapunov diffusivity (color), with time-mean streamlines overplotted as black contours.

weaker jet regions of the Northern Hemisphere, which is consistent with the stretching rate calculations of Scott et al. (2003).

Comparing the results with Fig. $3 \mathrm{c}$ of Hitchman and Huesmann (2007), it can be seen that the barriers are closely associated with regions of strong potential vorticity gradients and with mixing regions with Rossby wave breaking (see also Strong and Magnusdottir 2008). Chen (1995) and Postel and Hitchman (1999) suggest that the mixing regions in the Southern Hemisphere may be associated with the subtropical highs of the summer monsoon circulations, and it can be seen here that there is a clear correspondence between the regions of strong mixing and deviations from zonal symmetry in the streamlines. In the tropics, although the time-mean winds are predominantly easterly, there are two regions of significant westerlies during boreal winter in the eastern Pacific and eastern Atlantic. In the presence of stronger equatorial westerlies, there is also stronger diffluence in the jet exit region in the Northern Hemisphere (Waugh and Polvani 2000). This wind structure allows for mixing in the jet exit regions and the propagation of waves in "westerly ducts" from the Northern
Hemisphere deep into the tropics and the even into the Southern Hemisphere (Tomas and Webster 1994; Waugh and Polvani 2000). Intrusions of stratospheric air into the troposphere of this type likely contribute to the regions of enhanced mixing in the tropics and subtropics of the eastern Pacific and eastern Atlantic.

At the equator, a very weak barrier exists over the Indian Ocean and western Pacific, and mixing regions are observed over the eastern Pacific and Atlantic. Part I indicated that the correlation between eddy diffusivity and modified Lyapunov exponents were rather weak in the tropical region $\left( \pm 15^{\circ}\right)$. A number of potential reasons for this were discussed in Part I. Caution therefore needs to be taken in interpreting the Lyapunov diffusivity results in this region.

Next, we consider the March-May (MAM) average (Fig. 1b), which is characterized by zonally extended minima in correspondence of the subtropical jets with less longitudinal variability. In the Northern Hemisphere, there is weak mixing throughout the high latitudes. The subtropical jets act as barriers to transport, although they are noticeably weaker barriers than in the winter season, particularly over Asia. There remains 

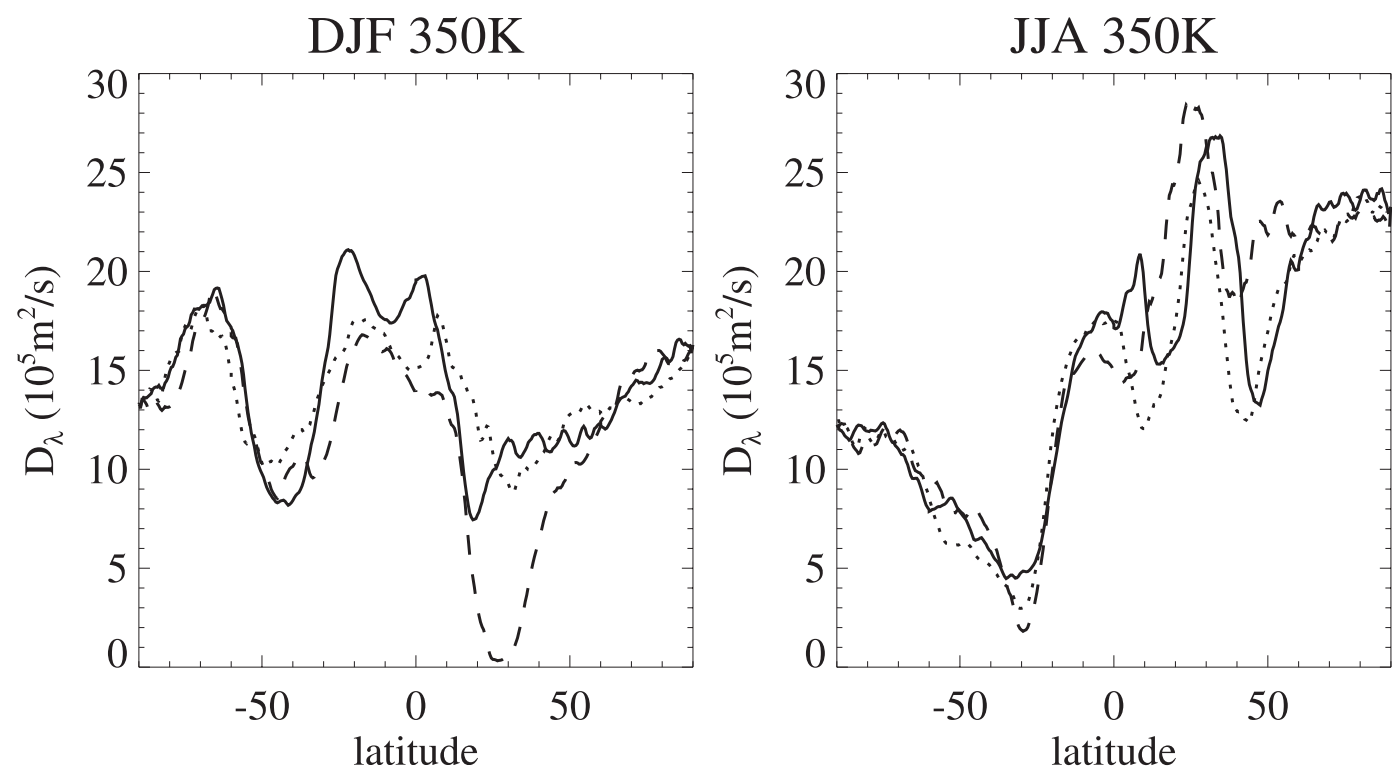

FIG. 2. Lyapunov diffusivity at specific longitudes: (left) for DJF at $25^{\circ}-30^{\circ} \mathrm{W}$ (solid), $130^{\circ}-135^{\circ} \mathrm{W}$ (dotted), and $115^{\circ}-120^{\circ} \mathrm{E}$ (dashed) and (right) for JJA at $45^{\circ}-50^{\circ} \mathrm{W}$ (solid), $55^{\circ}-60^{\circ} \mathrm{E}$ (dotted), and $115^{\circ}-120^{\circ} \mathrm{E}$ (dashed).

a region of enhanced mixing in the eastern Atlantic and across Southern Europe into Asia. The region of mixing over the eastern Pacific at the exit of the Pacific jet is stronger than in DJF, and it is bounded to the south and north by weak barriers. This may explain the springtime ozone mixing ratios from in situ aircraft measurements in this region described by Ray et al. (2004), and it may represent the existence of an eddy-driven jet poleward of the subtropical jet (Lee and Kim 2003). At the equator, there is a weak barrier that is centered in Southeast Asia, extends across the Indian Ocean and western Pacific, and weakens in the eastern Pacific and Atlantic in the regions of the westerly ducts. In the Southern Hemisphere, mixing follows a mostly zonal pattern, with a barrier at the subtropical jet and a subpolar band of mixing around the coast of Antarctica, maximizing in the vicinity of the Antarctic peninsula.

In June-August (JJA; Fig. 1c), there is strong mixing throughout much of the Northern Hemisphere and very weak mixing throughout much of the Southern Hemisphere (see also Fig. 2). There is a near-continuous band of enhanced mixing centered on approximately $30^{\circ} \mathrm{N}$, extending through the Tibetan high, across the Pacific, over the United States, and into the Atlantic. The regions of strongest mixing in this band are centered on the far western sides of the Pacific and Atlantic basins, slightly to the west of maxima in Rossby wave breaking (Postel and Hitchman 1999). The mixing observed in the Northern Hemisphere is of the greatest magnitude of either hemisphere in any season. This is in agreement with the Rossby wave-breaking statistics of Postel and
Hitchman (1999). They found a clear summertime predominance for Rossby wave breaking in both hemispheres, with approximately twice as many tropopause folds in the Northern Hemisphere than in the Southern Hemisphere. This also agrees with the results from tracer-tracer correlations in Hoor et al. (2002).

In the Northern Hemisphere, the weak mixing associated with the subtropical jet is located poleward of its DJF position, centered on about $40^{\circ}-50^{\circ} \mathrm{N}$. The diffusivities are typically $1 \times 10^{6}-1.5 \times 10^{6} \mathrm{~m}^{2} \mathrm{~s}^{-1}$ or more, except for a region of a stronger barrier over central Asia from approximately $20^{\circ}-100^{\circ} \mathrm{E}$, where the zonal winds of the subtropical jets are strongest on the northern branch of Asian monsoon anticyclone and in the western Atlantic (dotted and solid lines in Fig. 2). The diffusivity minima are in the vicinity of Turkey and Afghanistan, where Sprenger et al. (2003) find maxima in stratosphereto-troposphere and troposphere-to-stratosphere mass flux (based on three-dimensional trajectory calculations) in association with shallow and medium-depth tropopause folds. This serves as a reminder that the isentropic transport considered here is only one component of stratosphere-troposphere exchange (Holton et al. 1995).

In the Southern Hemisphere, there is an indication of two barriers: one associated with the subtropical jet, with the strongest barrier located over the Indian Ocean and western Pacific at about $30^{\circ} \mathrm{S}$, and the other associated with the polar jet at $60^{\circ} \mathrm{S}$ (see also Fig. 2). For most longitudes, these barriers are stronger (with diffusivities of $5 \times 10^{5} \mathrm{~m}^{2} \mathrm{~s}^{-1}$ and less) than the Southern Hemisphere subtropical barrier in DJF. This modulation of the 
strength of the barrier at the subtropical jet is consistent with the stretching rate calculations of Scott and Cammas (2002); although the winter troposphere is dynamically more active than the summer hemisphere, repeated studies (e.g., Scott and Cammas 2002) have indicated the winter subtropical jet acts as a stronger barrier, especially in the Northern Hemisphere, but also in the Southern Hemisphere. At high latitudes in the Southern Hemisphere, the mixing is considerably weaker than in DJF, which is consistent with a lower wave-breaking frequency Berrisford et al. (2007).

Comparing Figs. 1c and 3d of Hitchman and Huesmann (2007; see also Postel and Hitchman 1999), it can be seen that (i) the regions of strongest PV gradients in the subtropics correspond to barriers to mixing; (ii) strong mixing in the northern high latitudes corresponds to a region of significant Rossby wave breaking; and (iii) some of the regions of enhanced mixing in the subtropics, namely in the central Pacific and western Atlantic, corresponds to regions of enhanced Rossby wave breaking associated with the Asian and American monsoon circulations (Postel and Hitchman 1999). Tracer calculations (Chen 1995) and particle trajectory studies (Postel and Hitchman 1999) have indicated that significant stratosphere-to-troposphere exchange occurs adjacent to and downstream of these monsoon anticyclones in the region of enhanced Rossby wave breaking (Dunkerton 1995) and that the stratospheric tracer is then mixed throughout the tropics. Other studies (Dethof et al. 1999) have also indicated that strong mixing is associated with the monsoon circulations. Enhanced mixing throughout much of the Northern Hemisphere subtropics is also indicated from analysis of tracer data from satellite observations (Patmore and Toumi 2006). Moreover, the Lyapunov diffusivities indicate that the strong mixing associated with the Northern Hemisphere summer monsoons is bounded to the north and south by barriers at approximately $45^{\circ}$ and $10^{\circ} \mathrm{N}$ (see also Fig. 2). This is in agreement with the chemical isolation of the Asian monsoon anticyclone that was deduced from carbon monoxide observations by Park et al. (2008).

In September-November (SON; Fig. 1d), changes in the monsoon circulations are reflected in changes in the mixing patterns. Mixing is significantly reduced over Asia, although there are still regions of enhanced mixing across the Pacific through to the Atlantic and over Central Africa.

\section{Interannual variability}

In this section we investigate some aspects of the interannual variability of the mixing and transport structure. We first consider variations associated with El Niño-
Southern Oscillation (ENSO), and then we consider the North Atlantic Oscillation (NAO) and the Antarctic Oscillation (AAO).

\section{a. El Niño-Southern Oscillation}

Because we have already observed there to be a strong annual cycle in the transport and mixing of this UTLS region, we now consider the influence of ENSO on the transport and mixing structure of the seasons separately. Figure 3 presents the Lyapunov diffusivity for DecemberFebruary (left) and June-August (right) with a composite of La Niña years (negative phase) in the top panels and El Niño years (positive phase) in the middle panels. ${ }^{2}$ These are to be compared with the means for the entire period 1981-2001 in Fig. 1. It can be seen from Fig. 3 that the ENSO influence is indeed strongly dependent on season.

Figure 3 (bottom) presents the linear correlation between the Lyapunov diffusivity and the Niño-3.4 sea surface temperature index (Rayner et al. 2003; other ENSO indices produced very similar results). As one might expect, the greatest correlation is in the tropics and subtropics of the Pacific.

In DJF, in the Northern Hemisphere, a very strong barrier exists at all subtropical longitudes in the positive phase. The subtropical jet in the Pacific sector is stronger and more zonal in the positive phase; accordingly, the barrier extends farther into the eastern Pacific, which is in agreement with the results of Scott and Cammas (2002; see also Scott et al. 2003). In addition, there is stronger than usual mixing immediately poleward of the jet (e.g., over the central United States at about $40^{\circ} \mathrm{N}$, $\left.90^{\circ} \mathrm{W}\right)$. In the Southern Hemisphere, the barrier at the subtropical jet is also stronger in the positive phase (negative correlation); also in there is a band of positive correlation at about $60^{\circ} \mathrm{S}$ at all longitudes. This banded structure in the correlations is suggestive of an equatorward shift and a strengthening of the barriers at the subtropical jet during the positive phase (Shapiro et al. 2001). Indeed, in both hemispheres, it is clear from the streamlines that the jets are stronger and more zonal in the positive phase.

Previous studies (Shapiro et al. 2001; Martius et al. 2007) have shown there to be an increase in occurrence of cyclonic-type (LC2) wave-breaking events during

\footnotetext{
${ }^{2}$ We remind the reader that some caution is needed in the interpretation of the results in the region $\pm 15^{\circ}$, because rather poor agreement was found in this region between the effective diffusivity and the modified Lyapunov diffusivities. We note, as discussed in this section, that the results we find in the tropics are nevertheless broadly consistent with existing observational/modeling studies.
} 
DJF (negative)

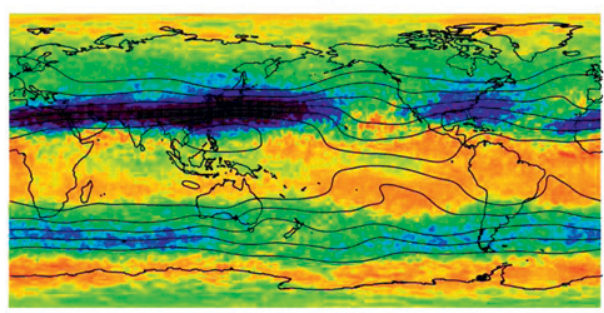

DJF (positive)

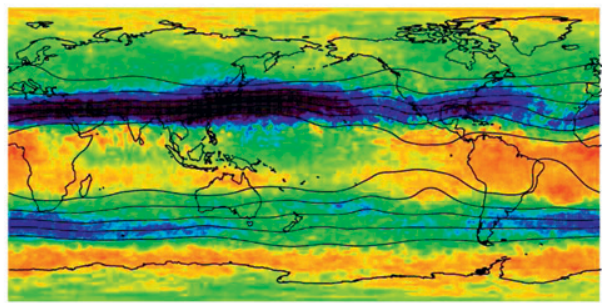

JJA (negative)

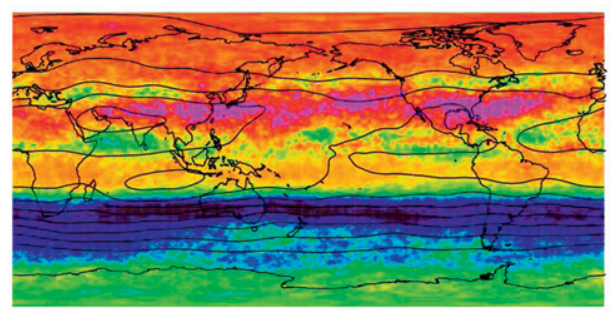

JJA (positive)

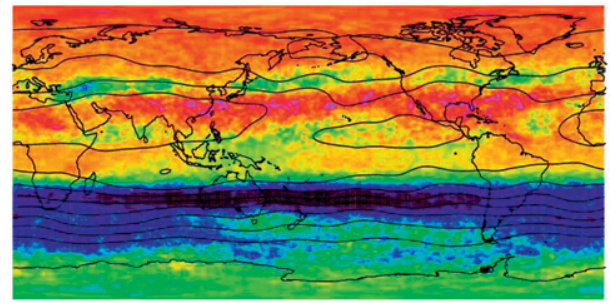

$90 \quad 180$

$-90$

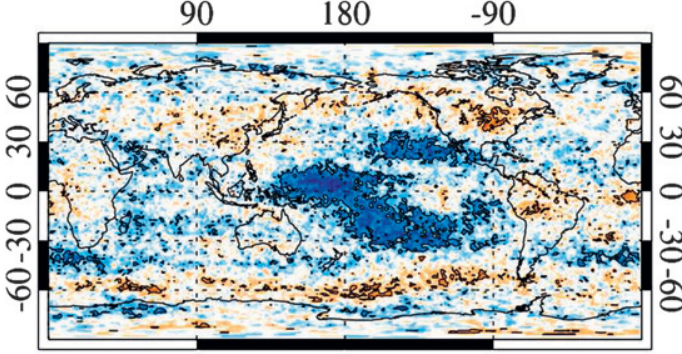

90

180

$-90$
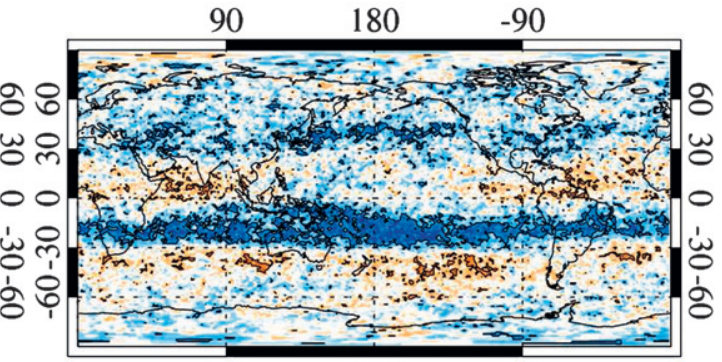

90

180

$-90$

FIG. 3. Composite of Lyapunov diffusivities $D_{\lambda}$ for the strongest (top) negative and (middle) positive phase months of ENSO for (left) DJF and (right) JJA. The color scale is as in Fig. 1. The composite streamlines are overplotted. (bottom) The correlation between the Niño-3.4 SST index and the seasonal Lyapunov diffusivities for the period 1981-2001. The black contours mark regions of greater than $95 \%$ statistical significance.

the positive phase and of anticyclonic-type (LC1) events during the negative phase of ENSO. LC2-type events are associated with poleward breaking and produce cut-off cyclones at high latitudes; LC1-type events are instead associated with equatorward breaking. These differences in the latitudes at which mixing occurs may contribute toward the greater mixing poleward of the jet over North America and the western Atlantic in the positive phase. Note, however, that recent studies (Polvani and Esler 2007) have indicated that the amount of transport from the stratosphere to the troposphere is significantly larger for LC2, and hence in the positive phase, than for LC1.

There are negative correlations in the subtropics over the eastern Pacific and at the equator in the western Pacific, in the vicinity of the date line. These correspond to regions of stronger barriers and/or reduced mixing in the positive phase of ENSO. In the central Pacific, there is noticeably less mixing in the negative phase just north of the equator, which is coincident with the strongest westerly winds, but significantly more mixing to the south, in the Southern Hemisphere subtropics. This pattern is consistent with the observation that, in the positive phase, the region of upper-tropospheric mean easterlies over the tropical western Pacific expands eastward past the date line, and the upper-tropospheric mean westerly duct over the tropical eastern Pacific is substantially weakened (Matthews and Kiladis 1999; Waugh and Polvani 2000; Shapiro et al. 2001; Waugh and Funatsu 2003). This region is just to the west of a region of enhanced transient convection in the negative phase (see Matthews and Kiladis 1999, their Fig. 5), which is 
likely associated with the enhanced equatorward wave propagation (see also Waugh and Funatsu 2003).

Across South America and in the Atlantic sector, there is a region of positive correlation in the Southern Hemisphere tropics, with a negative correlation in the subtropics. This corresponds to enhanced mixing in the tropics in the positive phase, which extends westward over northern South America and eastward toward Central Africa. This is consistent with the suggestion of Tomas and Webster (1994) that, in the positive phase, an increase in the upper-tropospheric mean westerlies in the eastern Atlantic region (Arkin and Webster 1985) leads an enhancement of interhemispheric wave propagation in the westerly duct in boreal winter-spring.

In JJA, the influence of ENSO is observed at almost all longitudes in the tropics and subtropics. In the Southern Hemisphere, there is a significant strengthening and an equatorward shift of the barrier at the subtropical jet at all longitudes.

ENSO is known to have a major influence on the variability of the Asian-Australian monsoon system (Lau and Nath 2006; Wang et al. 2003; Chou et al. 2003; Wang et al. 2000). Low-level circulation anomalies in the south Indian Ocean and western North Pacific are thought to be associated with a remote atmospheric Rossby wave response to the altered condensational heating over the equatorial western Pacific during ENSO events, in combination with local atmosphereocean interactions. The timing of these low-level circulation anomalies appears to be linked to the developing and decaying phases of ENSO. Overall, however, the entire monsoon system tends to weaken during the positive phase and strengthen during the negative phase, with the greatest weakening being during the fall of El Niño development (Wang et al. 2003). Consistent with this, we observe in Fig. 3 an enhancement of the mixing structure associated with the Asian-Australian monsoon system in the negative phase. The mixing is observed to be both greater in magnitude in the negative phase and also to extend farther eastward over into the Pacific and westward over India.

A strong correlation is also observed between ENSO and mixing regions in JJA in the eastern Pacific and over North America into the western Atlantic in the Northern Hemisphere subtropics. This mixing is observed to be stronger during the negative phase and confined to the Northern Hemisphere by a stronger barrier at the equator, which is linked to a westerly wind anomaly.

\section{b. North Atlantic Oscillation}

The NAO is the main mode of variability for the climate of the North Atlantic and western Europe. This mode is determined by the intensities of a low pressure system centered over Iceland and a high pressure system centered over the Azores. These two pressure anomalies control the strength and direction of the westerly winds associated with the subtropical jet. A positive phase (intensification of the difference between the Icelandic low and the Azores high) corresponds to stronger westerlies that are directed more to the north of Europe, whereas a negative phase results in weaker westerlies, which are tracked toward the Mediterranean. In Fig. 4 (left panel), we consider wintertime mixing for composite years of positive and negative NAO phase (station-based index from the Climate Research Unit).

In the negative phase, the mixing is reduced throughout most the regions from $20^{\circ}$ to $60^{\circ} \mathrm{N}$, with the strongest barrier being centered on the subtropical jet at approximately $35^{\circ} \mathrm{N}$. During the positive phase, there are weak barriers following the jet across the Atlantic in two branches: one toward Northern Europe and the other toward North Africa. The southern branch is equatorward of its position during the negative phase. Significant mixing is observed between these two branches. This pattern of variability of transport and mixing is consistent with an intensification of wave activity during the positive phase (Limpasuvan and Hartman 1999); Rossby waves being refracted equatorward when the jet is displaced poleward (Limpasuvan and Hartman 2000); and a tendency for more anticyclonic (LC1) wave breaking (Martius et al. 2007; Benedict et al. 2004), as well as more poleward, cyclonic wave breaking during the negative phase (Woollings et al. 2008). It may also play a role in the evolution of the NAO itself (DeWeaver and Nigam 2000; Benedict et al. 2004; Franzke et al. 2004; Martius et al. 2007; Rivière and Orlanski 2007; Woollings et al. 2008; Strong and Magnusdottir 2008).

Figure 4 (bottom) shows the correlation between the Lyapunov diffusivity and the NAO index. The pattern of positive correlation at $\sim 30^{\circ} \mathrm{N}$ and negative correlation at $\sim 60^{\circ} \mathrm{N}$ (which corresponds to a poleward shift in the location of the subtropical jet in the positive phase) is seen at all longitudes but is only statistically significant in the Atlantic sector plotted in Fig. 4.

\section{c. Antarctic Oscillation}

The AAO is the leading mode of variability in the Southern Hemisphere, and it is characterized by geopotential height perturbations throughout the troposphere and stratosphere of opposing signs in the polar cap region and the midlatitudes (Thompson and Wallace 2000). An index of this oscillation is obtained by taking the empirical orthogonal functions of monthly mean 1000-hPa height anomalies poleward of $20^{\circ}$ latitude. Figure 4 (right) considers the variability of the transport and mixing structure associated with this oscillation. 


\section{DJF (NAO-)}

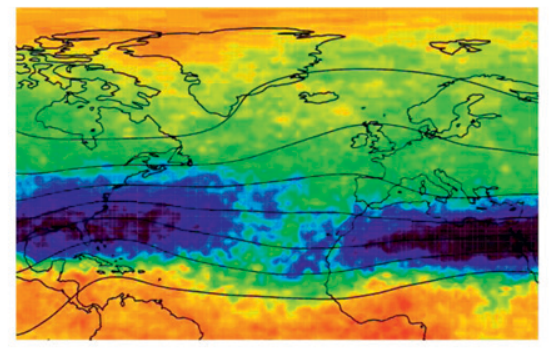

DJF (NAO+)
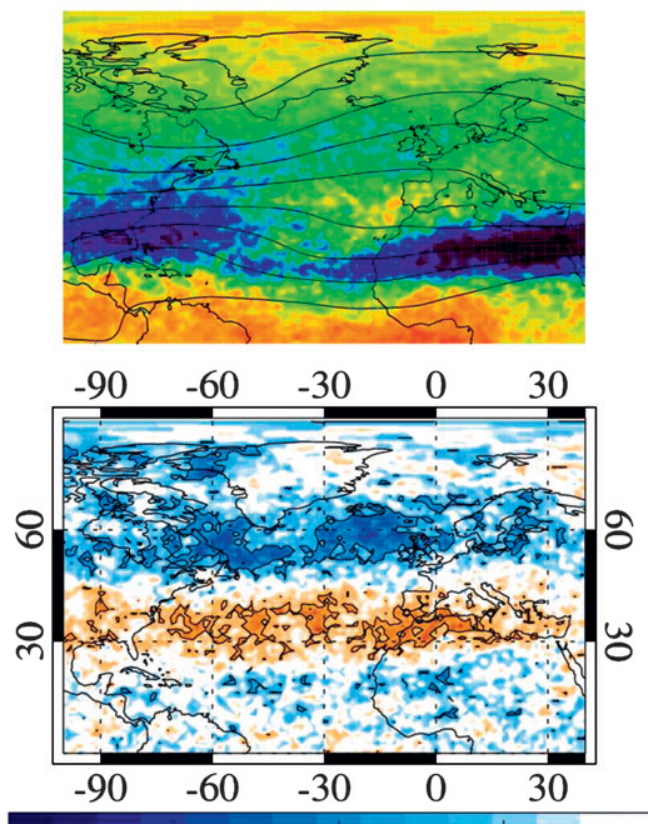

$\begin{array}{lll}-0.75 & -0.5 & -0.25\end{array}$

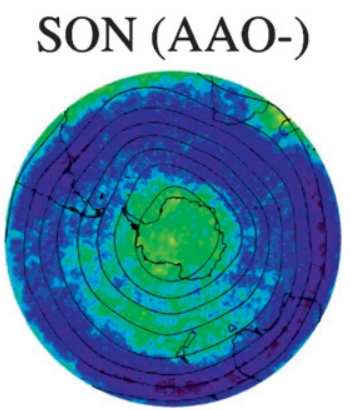

\section{$\mathrm{SON}(\mathrm{AAO}+)$}
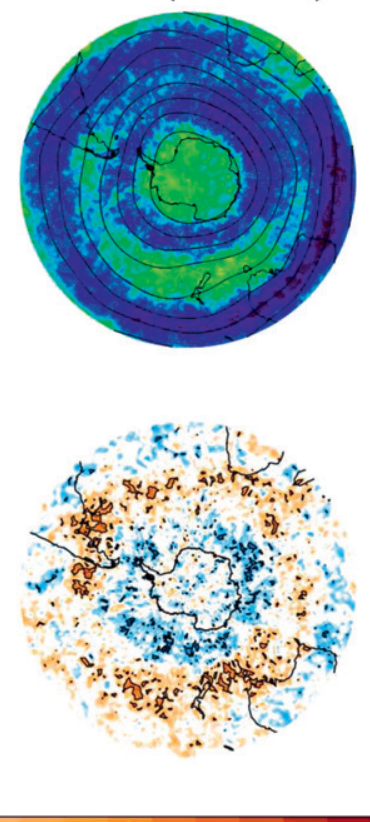

\section{$0.5 \quad 0.75$}

FIG. 4. Composite of $D_{\lambda}$ for the strongest (top) negative and (middle) positive phase months of (left) NAO (DJF for the northern Atlantic) and (right) AAO (SON for the Southern Hemisphere). The color scale is as in Fig. 1. The composite streamlines are overplotted. (bottom) The correlation between the index and the annual $D_{\lambda}$ over the period 1981-2001 for (left) NAO in DJF and (right) AAO in SON. The black contours mark regions of greater than $95 \%$ statistical significance.

Significant correlation between the Lyapunov diffusivities and the AAO is observed in all seasons; here, we present the results for September-November (the time series were detrended before the correlations were calculated). In the positive phase, the general pattern in each season is a weaker barrier at the subtropical jet, which is shifted slightly poleward, and weaker mixing over the polar cap. The exception to this pattern can be seen in the western Pacific sector for SON, where the barrier at the subtropical jet is narrower but stronger in the positive phase. This may be due to an intensification of the PV gradients associated with the jet by the enhanced mixing region poleward of the jet (in analogy with the sharpening of the PV gradients at the stratospheric vortex edge by mixing induced by Rossby wave breaking in the surf zone).

Also visible in the figure for the positive phase in SON is a second barrier associated with the polar jet. This barrier is connected to the barrier at the vortex edge in the stratosphere (Haynes and Shuckburgh 2000b). This split jet structure over New Zealand is a known feature of the Southern Hemisphere circulation, and the mixing region between the two jets corresponds to a region of transient eddy activity (Marshall and King 1998). Consistent with our results, Berrisford et al. (2007) found a significant correlation between the AAO and wavebreaking frequency in this region. 
Observations indicate that there has been a recent shift toward a more positive phase of the AAO (Thompson and Solomon 2002), and the debate is ongoing as to future predictions (Son et al. 2008). As noted by Son et al. (2008), the circulation has a number of important consequences that directly affect, among other things, surface temperature, sea ice extent, wind-driven ocean circulations, and the exchange of carbon dioxide between atmosphere and ocean. Here, it can be seen that it also influences the transport of chemical species into and out of the subvortex region. Model results (Lee et al. 2002) indicate that significant in situ ozone depletion occurs within the subvortex region (accounting for over a quarter of the total depletion). Whether this air is transported equatorward will have an important influence on the chemical composition and radiative properties of the midlatitude lowermost stratosphere and upper troposphere.

\section{Discussion}

In this paper, we have investigated the seasonal and interannual variability of the transport and mixing structure of the UTLS using the new Lyapunov diffusivity diagnostic. A strong seasonal cycle has been observed in the strength of the barriers at the subtropical jets and in mixing regions associated with the monsoon circulations. The strongest mixing is observed to the east of the monsoon anticyclones in the Northern Hemisphere summer. The strongest barriers are observed associated with the strongest westerly velocities of the subtropical jet over Asia in Northern Hemisphere winter.

Longitudinal variability has been shown to be influenced by modes of atmospheric variability. ENSO strongly influences the strength and position of the barriers at the subtropical jets, and it also modulates the strength of mixing associated with the monsoons. In addition, its effect on the westerly ducts in the subtropics influences mixing in the equatorial eastern Pacific and Atlantic. The changes to the subtropical jet associated with the North Atlantic Oscillation and the changes to the subtropical and polar jets associated with the Antarctic Oscillation both influence the local mixing and transport structure.

The transport and mixing structure revealed by the Lyapunov diffusivity is largely in agreement with existing results based on model studies and observational data. Many of the regions of high values of Lyapunov diffusivity coincide with geographical locations that have been determined on the basis of analysis of potential vorticity fields to exhibit frequent intrusion events (e.g., Tomas and Webster 1994; Waugh and Polvani 2000) or statistically large Rossby wave breaking (e.g., Postel and Hitchman
1999; Hitchman and Huesmann 2007; Berrisford et al. 2007). Similarly, the results are generally consistent with regional observations of chemical species from aircraft campaigns (e.g., Ziereis et al. 2000; Ray et al. 2004; Spackman et al. 2007). The advantage of the Lyapunov diffusivity is that it can be applied globally, and it provides a quantitative measure of mixing in units of diffusion. This allows for the robust comparison of different geographical regions and different time periods.

The accurate representation of transport and mixing in the UTLS in chemistry-climate models is essential for the correct prediction of future climate change, because it influences the distribution of radiatively significant chemical species. As a consequence, new diagnostics are being sought to evaluate model performance (e.g., Pan et al. 2007). One potential application of the Lyapunov diffusivity is as a tool for the comparison of the transport and mixing properties of velocities coming from different chemistry-climate models and different analyzed wind datasets.

Acknowledgments. F.d'O. has been supported during this research by the Marie-Curie fellowship (024717DEMETRA). EFS is supported by a NERC postdoctoral fellowship. We also acknowledge the support of the EU integrated project SCOUT (Contract 505390GOCECT-2004).

\section{REFERENCES}

Arkin, P. A., and P. J. Webster, 1985: Annual and interannual variability of tropical-extratropical interaction: An empirical study. Mon. Wea. Rev., 113, 1510-1523.

Benedict, J. J., S. Lee, and S. B. Feldstein, 2004: Synoptic view of the North Atlantic Oscillation. J. Atmos. Sci., 61, 121-144.

Berrisford, P., B. J. Hoskins, and E. Tyrlis, 2007: Blocking and Rossby wave breaking on the dynamical tropopause in the Southern Hemisphere. J. Atmos. Sci., 64, 2881-2898.

Chen, P., 1995: Isentropic cross-tropopause mass exchange in the extratropics. J. Geophys. Res., 100, 16 661-16 673.

Chou, C., J.-Y. Tu, and J.-Y. Yu, 2003: Interannual variability of the western North Pacific summer monsoon: Differences between ENSO and non-ENSO years. J. Climate, 16, 2275-2287.

Dethof, A., A. O’Neill, J. M. Slingo, and H. G. J. Smit, 1999: A mechanism for moistening the lower stratosphere involving the Asian summer monsoon. Quart. J. Roy. Meteor. Soc., 125B, 1079-1106.

DeWeaver, E., and S. Nigam, 2000: Do stationary waves drive the zonal-mean jet anomalies of the northern winter? J. Climate, 13, 2160-2176.

d’Ovidio, F., V. Fernández, E. Hernández-García, and C. López, 2004: Mixing structures in the Mediterranean Sea from finitesize Lyapunov exponents. Geophys. Res. Lett., 31, L17203, doi:10.1029/2004GL020328.

, E. Shuckburgh, and B. Legras, 2009: Local mixing events in the upper troposphere and lower stratosphere. Part I: Detection with the Lyapunov diffusivity. J. Atmos. Sci., 66, 3678-3694. 
Dunkerton, T. J., 1995: Evidence of meridional motion in the summer lower stratosphere adjacent to monsoon regions. J. Geophys. Res., 100, 16 675-16 688.

Franzke, C., S. Lee, and S. B. Feldstein, 2004: Is the North Atlantic Oscillation a breaking wave? J. Atmos. Sci., 61, 145-160.

Haynes, P., and E. Shuckburgh, 2000a: Effective diffusivity as a diagnostic of atmospheric transport. 1. Stratosphere. J. Geophys. Res., 105 (D18), 22 777-22 794.

— mospheric transport. 2. Troposphere and lower stratosphere. J. Geophys. Res., 105 (D18), 22 795-22 810.

- D. A. Poet, and E. F. Shuckburgh, 2007: Transport and mixing in kinematic and dynamically consistent flows. $J$. Atmos. Sci., 64, 3640-3651.

Hegglin, M. I., and Coauthors, 2006: Measurements of NO, $\mathrm{NO}_{y}$, $\mathrm{N}_{2} \mathrm{O}$ and $\mathrm{O}_{3}$ during spurt: Implications for transport and chemistry in the lowermost stratosphere. Atmos. Chem. Phys., 6 , 1331-1350.

—_, and Coauthors, 2008: Validation of ACE-FTS satellite data in the upper troposphere/lower stratosphere (UTLS) using noncoincident measurements. Atmos. Chem. Phys., 8, 1483-1499.

Hitchman, M. H., and A. S. Huesmann, 2007: A seasonal climatology of Rossby wave breaking in the 320-2000-K layer. J. Atmos. Sci., 64, 1922-1940.

Holton, J. R., P. H. Haynes, M. E. McIntyre, A. R. Douglass, R. B. Rood, and L. Pfister, 1995: Stratosphere-troposphere exchange. Rev. Geophys., 33, 403-439.

Hoor, P., H. Fischer, L. Lange, J. Lelieveld, and D. Brunner, 2002: Seasonal variations of a mixing layer in the lowermost stratosphere as identified by the $\mathrm{CO}-\mathrm{O}_{3}$ correlation from in situ measurements. J. Geophys. Res., 107, 4044, doi:10.1029/ 2000JD000289.

Hoskins, B. J., 1991: Towards a PV- $\theta$ view of the general circulation. Tellus, 43, 27-35.

_ , and P. J. Valdes, 1990: On the existence of storm-tracks. J. Atmos. Sci., 47, 1854-1864.

Joseph, B., and B. Legras, 2002: Relation between kinematic boundaries, stirring, and barriers for the Antarctic polar vortex. J. Atmos. Sci., 59, 1198-1212.

Koh, T., and B. Legras, 2002: Hyperbolic lines and the stratospheric polar vortex. Chaos, 12, 382-394.

Lau, N.-C., and M. Nath, 2006: ENSO modulation of the interannual and intraseasonal variability of the East Asian monsoon-A model study. J. Climate, 19, 4508-4530.

Lee, A. M., R. L. Jones, I. Kilbane-Dawe, and J. A. Pyle, 2002: Diagnosing ozone loss in the extratropical lower stratosphere. J. Geophys. Res., 107, 4110, doi:10.1029/2001JD000538.

Lee, S., and H. K. Kim, 2003: The dynamical relationship between subtropical and eddy-driven jets. J. Atmos. Sci., 60, 1490-1503.

Limpasuvan, V., and D. L. Hartman, 1999: Eddies and the annular modes of climate variability. Geophys. Res. Lett., 26, 3133-3136.

$\longrightarrow$, and 2 2000: Wave-maintained annular modes of climate variability. J. Climate, 13, 4414-4429.

Marshall, G. J., and J. C. King, 1998: Southern Hemisphere circulation anomalies associated with extreme Antarctic peninsula winter temperatures. Geophys. Res. Lett., 25, 2437-2440.

Martius, O., C. Schwierz, and H. C. Davies, 2007: Breaking waves at the tropopause in the wintertime Northern Hemisphere: Climatological analyses of the orientation and the theoretical LC1/2 classification. J. Atmos. Sci., 64, 2576-2592.

Matthews, A. J., and G. N. Kiladis, 1999: Interactions between ENSO, transient circulation, and tropical convection over the Pacific. J. Climate, 12, 3062-3086.
McIntyre, M. E., 2008: Potential-vorticity inversion and the waveturbulence jigsaw: Some recent clarifications. Adv. Geosci., 15, $47-56$.

Nakamura, N., 1996: Two-dimensional mixing, edge formation, and permeability diagnosed in an area coordinate. J. Atmos. Sci., 53, 1524-1537.

Pan, L. L., J. C. Wei, D. E. Kinnison, R. R. Garcia, D. J. Wuebbles, and G. P. Brasseur, 2007: A set of diagnostics for evaluating chemistry-climate models in the extratropical tropopause region. J. Geophys. Res., 112, D09316, doi:10.1029/2006JD007792.

Park, M., W. J. Randel, L. K. Emmons, P. F. Bernath, K. A. Walker, and C. D. Boone, 2008: Chemical isolation in the Asian monsoon anticyclone observed in Atmospheric Chemistry Experiment (ACE-FTS) data. Atmos. Chem. Phys., 8, 757-764.

Patmore, N., and R. Toumi, 2006: An entropy-based measure of mixing at the tropopause. Quart. J. Roy. Meteor. Soc., 132, 1949-1967.

Polvani, L. M., and J. G. Esler, 2007: Transport and mixing of chemical air masses in idealized baroclinic life cycles. J. Geophys. Res., 112, D23102, doi:10.1029/2007JD008555.

Postel, G. A., and M. H. Hitchman, 1999: A climatology of Rossby wave breaking along the subtropical tropopause. J. Atmos. Sci., 56, 359-373.

Ray, E. A., K. H. Rosenlof, E. Richard, D. Parrish, and R. Jakoubek, 2004: Distributions of ozone in the region of the subtropical jet: An analysis of in situ aircraft measurements. J. Geophys. Res., 109, D08106, doi:10.1029/2003JD004143.

Rayner, N. A., D. E. Parker, E. B. Horton, C. K. Folland, L. V. Alexander, D. P. Rowell, E. C. Kent, and A. Kaplan, 2003: Global analyses of sea surface temperature, sea ice, and night marine air temperature since the late nineteenth century. J. Geophys. Res., 108, 4407, doi:10.1029/2002JD002670.

Rivière, G., and I. Orlanski, 2007: Characteristics of the Atlantic storm-track eddy activity and its relation with the North Atlantic Oscillation. J. Atmos. Sci., 64, 241-266.

Scott, R. K., and J.-P. Cammas, 2002: Wave breaking and mixing at the subtropical tropopause. J. Atmos. Sci., 59, 2347-2361.

E. F. Shuckburgh, J.-P. Cammas, and B. Legras, 2003: Stretching rates and equivalent length near the tropopause. J. Geophys. Res., 108, 4394, doi:10.1029/2002JD002988.

Shapiro, M. A., H. Wernli, N. A. Bond, and R. Langland, 2001: The influence of the 1997-1999 ENSO on extratropical baroclinic life cycles over the eastern North Pacific. Quart. J. Roy. Meteor. Soc., 127, 331-342.

Shuckburgh, E., and P. Haynes, 2003: Diagnosing transport and mixing using a tracer-based coordinate system. Phys. Fluids, 15, 3342-3357.

, H. Jones, J. Marshall, and C. Hill, 2009a: Robustness of effective diffusivity diagnostic in oceanic flows. J. Phys. Oceanogr., 39, 1993-2009.

$\ldots, \ldots, \ldots$, and $-\ldots 09 \mathrm{~b}$ : Understanding the regional variability of eddy diffusivity in the Pacific sector of the Southern Ocean. J. Phys. Oceanogr., 39, 2011-2023.

Son, S.-W., and Coauthors, 2008: The impact of stratospheric ozone recovery on the southern hemisphere westerly jet. Science, 320, $1486-1489$.

Spackman, J. R., E. M. Weinstock, J. G. Anderson, D. F. Hurst, H.-J. Jost, and S. M. Schauffler, 2007: Aircraft observations of rapid meridional transport from the tropical tropopause layer into the lowermost stratosphere: Implications for midltitude ozone. J. Geophys. Res., 112, D12308, doi:10.1029/2006JD007618.

Sprenger, M., M. C. Maspoli, and H. Wernli, 2003: Tropopause folds and cross-tropopause exchange: A global investigation 
based upon ECMWF analyses for the time period March 2000 to February 2001. J. Geophys. Res., 108, 8518, doi:10.1029/ 2002JD002587.

Stohl, A., H. Wernli, P. James, M. Bourqui, C. Forster, M. A. Liniger, P. Seibert, and M. Sprenger, 2003: A new perspective of stratosphere-troposphere exchange. Bull. Amer. Meteor. Soc., 84, 1565-1573.

Strahan, S. E., B. N. Duncan, and P. Hoor, 2007: Observationally derived transport diagnostics for the lowermost stratosphere and their application to the GMI chemistry and transport model. Atmos. Chem. Phys., 7, 2435-2445.

Strong, C., and G. Magnusdottir, 2008: Tropospheric Rossby wave breaking and the NAO/NAM. J. Atmos. Sci., 65, 28612876.

Thompson, D. W. J., and J. M. Wallace, 2000: Annular modes in the extratropical circulation. Part I: Month-to-month variability. J. Climate, 13, 1000-1016.

— Hemisphere climate change. Science, 296, 895-899.

Tomas, R., and P. Webster, 1994: Horizontal and vertical structure of cross-equatorial wave propagation. J. Atmos. Sci., 51, $1417-1430$.
Uppala, S. M., and Coauthors, 2005: The ERA-40 Re-Analysis. Quart. J. Roy. Meteor. Soc., 131, 2961-3012.

Wang, B., R. Wu, and X. Fu, 2000: Pacific-East Asian teleconnection: How does ENSO affect East Asian climate? J. Climate, 13, 1517-1536.

,-- , and T. Li, 2003: Atmosphere-warm ocean interaction and its impacts on Asian-Australian monsoon variation. J. Climate, 16, 1195-1211.

Waugh, D. W., and L. M. Polvani, 2000: Climatology of intrusions into the tropical upper troposphere. Geophys. Res. Lett., 27, 3857-3860.

_ , and B. M. Funatsu, 2003: Intrusions into the tropical upper troposphere: Three-dimensional structure and accompanying ozone and OLR distributions. J. Atmos. Sci., 60, 637-653.

Woollings, T., B. Hoskins, M. Blackburn, and P. Berrisford, 2008: A new Rossby wave-braking interpretation of the North Atlantic Oscillation. J. Atmos. Sci., 65, 609-626.

Ziereis, H., H. Schlager, H. Fischer, C. Feigl, P. Hoor, R. Marquardt, and V. Wagner, 2000: Aircraft measurements of tracer correlations in the Arctic subvortex region during the Polar Stratospheric Aerosol Experiment (POLSTAR). J. Geophys. Res., 105, 24 305-24 313. 